\title{
Angular Correlations of Coincident Electron-Positron Pairs Produced in Heavy-Ion Collisions with Nuclear Time Delay
}

\author{
O. Graf, J. Reinhardt, B. Müller, and W. Greiner \\ Institut für Theoretische Physik, Universität Frankfurt, Postfach 111932, \\ D-6000 Frankfurt am Main 11, Federal Republic of Germany
}

G. Soff

Gesellschaft für Schwerionenforschung (GSI), Postfach 110552, D-6100 Darmstadt, Federal Republic of Germany

(Received 12 April 1988)

\begin{abstract}
We calculate angular correlations between coincident electron-positron pairs emitted in heavy-ion collisions with nuclear time delay. Special attention is directed to a comparison of supercritical and subcritical systems, where angular correlations of pairs produced in collisions of bare $U$ nuclei are found to alter their sign for nuclear delay times of the order of $2 \times 10^{-21} \mathrm{~s}$. This effect is shown to occur exclusively in supercritical systems, where spontaneous positron creation is active.
\end{abstract}

PACS numbers: $25.70 .-\mathrm{z}, 12.20 . \mathrm{Ds}, 34.50 . \mathrm{Fa}$

The process of spontaneous positron emission is intimately connected to the theory of quantum electrodynamics of strong fields. ${ }^{1}$ If two nuclei with combined charge $Z_{u}=Z_{1}+Z_{2} \geq 174$ are brought closely together, theory predicts a vacant $1 s \sigma$ state to dive into the lower continuum and to decay subsequently into adjacent positron states. ${ }^{1,2}$ Other predictions, such as productions of inner-shell holes, $\delta$ electrons, and positrons induced by the rapid variation of the electromagnetic field during the collision, have been studied in many heavy-ion scattering experiments, ${ }^{3-6}$ and their gross properties are well understood.

However, currently available experimental evidence cannot prove the spontaneous emission of positrons associated with the decay of the $1 s \sigma$ resonance. As consequence of the short time scale of the collision and the resulting Heisenberg broadening no qualitative signature is expected in the positron spectra, apart from a steep, but smooth increase in intensity with the nuclear charge $Z$. In supercritical collisions with time delay due to nuclear contact one expects that the spontaneous decay process is enhanced, eventually leading to the emergence of a peak structure in the positron spectrum. ${ }^{7,8}$ Unfortunately, rather long delay times of the order $10^{-20} \mathrm{~s}$ are required to obtain a clear signal. In the following we will therefore investigate the angular correlations of coincident electron-positron pairs created in heavy-ion collisions, which will be shown to provide further information on the pair-creation process, being particularly sensitive to the presence of spontaneous positron production.

We calculate the emission probability for electrons of energy $E_{e^{-}}$emitted into the direction $\mathbf{p}_{e}-/\left|\mathbf{p}_{e^{-}}\right|$in coincidence with positrons with energy $E_{e}+$ emitted in the direction $\mathbf{p}_{e}+/\left|\mathbf{p}_{e^{+}}\right|$. We employ the semiclassical formalism of coupled-channel equations. ${ }^{2}$ Electron screening is taken into account within the adiabatic HartreeFock-Slater approximation. ${ }^{9}$ In order to determine the propagation directions of emitted particles we transform the solutions of the time-dependent two-center Dirac problem from the spherical-basis representation with good total angular momentum to a basis set of Coulomb-distorted plane-wave states. ${ }^{2}$ Angular correlations should result from the interference of partial waves with different parity and angular momentum. ${ }^{10}$ We restrict our calculations to the monopole approximation, taking into account only the dominant angular momentum channels $s_{1 / 2}$ and $p_{1 / 2}(\kappa= \pm 1)$. This is expected to be justified, because as a result of the strong relativistic contraction of the electronic states in these channels their matrix elements are dominant over those connecting to higher $\kappa$ values. The good agreement of experimental data with predictions for single-emission probabilities validates this approximation. ${ }^{11,12}$ The final result for the number of $e^{+} e^{-}$pairs created in a collision summed over the helicities of the electron and positron is ${ }^{2,13}$

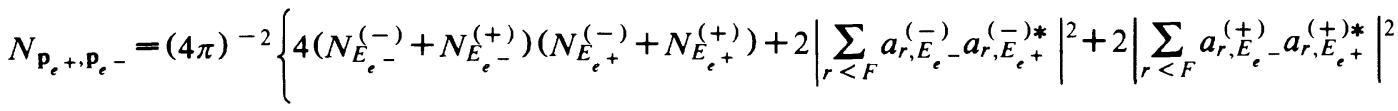

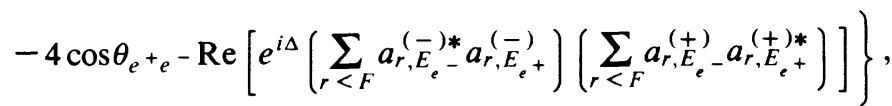

with the phase

$$
\Delta=\left(\delta_{E_{e^{+}}}^{(-)}-\delta_{E_{e^{+}}}^{(+)}\right)-\left(\delta_{E_{e^{-}}}^{(-)}-\delta_{E_{e}-}^{(+)}\right)
$$


and the singles electron and positron yields

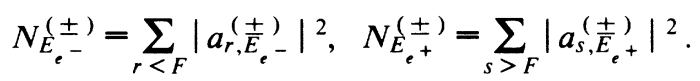

The quantities $a_{i f}$ are the asymptotic $(t \rightarrow \infty)$ amplitudes for a transition from state $i$ to state $f$ as obtained from the solution of the coupled-channel equations. The parameter $F$ (Fermi surface) which determines the scope of the summations defines the boundary between initially filled and initially vacant states. The superscript $( \pm)$ denotes the two considered angular momentum channels $\kappa= \pm 1 . \theta_{e}{ }^{+}{ }^{-}$describes the angle between the propagation directions of electron and positron, while $\delta_{E_{e} \pm}^{( \pm)}$ stands for the adiabatic phase shift due to the two-center potential.

The single phase shifts $\delta$ are found to vary strongly with the nuclear separation $R$ even at large $R$, where the monopole approximation becomes invalid. However, in both continua the phase difference between $\kappa=+1$ and $\kappa=-1$ states approaches a constant value ${ }^{13}$

$$
\delta_{e^{ \pm}}^{(-)}-\delta_{e^{ \pm}}^{(+)} \stackrel{R \rightarrow \infty}{\rightarrow} \pi / 2(\bmod \pi) .
$$

This has been verified in numerical solutions of the twocenter Dirac equation for continuum states. ${ }^{14}$ We introduce the following abbreviations for the terms entering the sum of Eq. (1):

$$
\begin{aligned}
N_{\mathbf{p}_{e}+\mathbf{p}_{e^{-}}} & =(4 \pi)^{-2}\left(S+C+A \cos \theta_{e^{+} e^{-}}\right) \\
& =a_{E_{e^{+}}, E_{e^{-}}}\left(1+a_{E_{e^{+}}, E_{e^{-}}} \cos \theta_{e^{+} e^{-}}\right) .
\end{aligned}
$$

We identify the term $S$ as statistical coincidences consisting of the product of the corresponding singleemission probabilities, while the terms $C$ and $A$ describe the isotropic and angular-dependent correlated pair emission. As a result of the property of the phases mentioned above the difference $D$ between $C$ and $A$ is found to depend in a simple way on the difference between the contributions of $\kappa=-1$ and $\kappa=+1$ channels:

$$
D=C-A=2\left|\sum_{r<F}\left(a_{r, E_{e^{-}}}^{(-)} a_{r, E_{e^{+}}}^{(-)}-a_{r, E_{e^{-}}}^{(+)} a_{r, E_{e^{+}}}^{(+)}\right)\right|^{2} .
$$

In subcritical systems we expect the difference $D$ to be approximately zero since excitations in the $\kappa=+1$ and $\kappa=-1$ channels are of similar size. This has been calculated in Ref. 13 for the collision system $\mathrm{Pb}-\mathrm{Pb}$ at $E_{\text {lab }}=5.7 \mathrm{MeV} /$ nucleon. In supercritical systems the difference $D$ between $\kappa=+1$ and $\kappa=-1$ contributions to correlated pair emission can be taken as a direct measure for spontaneous positron production in the $\kappa=-1$ channel. If $D$ exceeds $C$, the anisotropy coefficient $a^{1}=(C-D) /(C+S)$ will become negative. Unfortunately, in collisions of nuclei surrounded by electrons the large contribution of isotropic statistical coincidences $S$ leads to a strong relative suppression of angular correlations in coincident pair emission. For negative $a^{1}$ its magnitude will be $\left|a^{1}\right|<0.1$, so that the effect may be too small to be measurable. The origin of this suppression is the high ionization rate of inner-shell electrons which dominates the spectrum of emitted electrons and causes accidental coincidences. In principle this effect can be switched off by our studying a system devoid of bound electrons, i.e., the collision of two bare nuclei $(F=0)$. In ordinary collisions the contribution of the spontaneous mechanism to coincident $e^{+} e^{-}$pair production is at least a second-order process: production of a $K$-shell vacancy by electron emission, followed by production of a positron associated with filling of the vacant $1 s$ state. In collisions of bare nuclei the order of these steps is just reversed: First the $1 s$ vacancy is filled by an electron from the Dirac sea, i.e., by emission of a positron; then the $1 s$ electron is ejected into the continuum. Therefore in bare-ion collisions each emitted electron is necessarily correlated with a positron. Angular correlations can be used to obtain information on that fraction of pairs which has the $K$ shell as an intermediate state in the creation process.

We have compared elastic and time-delayed U-U and $\mathrm{Pb}-\mathrm{Pb}$ collisions of bare nuclei, applying a schematic trajectory model, ${ }^{8}$ which employs a fixed nuclear sticking time. In this case a significant difference $D$ between $\kappa=+1$ and $\kappa=-1$ appears in supercritical systems as well as in subcritical systems even for undelayed collisions. This is caused by the strong coupling of the negative-energy continuum to the vacant $1 s \sigma$ state, usually suppressed by the small production probability of $1 s \sigma$ holes during the nuclear approach. ${ }^{2}$ For increasing time delay $T$ the coefficient $a^{1}$ decreases further and can in fact become clearly negative for supercritical systems.

In order to use this effect as a signal for spontaneous positron production, we have to make sure that this phenomenon does not occur in subcritical systems. This is only partially fulfilled. Sharply defined reaction times lead to an interference pattern in the singles and coincident electron and positron spectra. ${ }^{15}$ In the vicinity of the oscillation minima $D$ can exceed $C$ [see Eqs. (1)-(3)], yielding a negative anisotropy coefficient $a^{1}$ also for subcritical systems. However, this effect is restricted to a narrow region in the spectrum and the phenomenon does not survive if we take into account a distribution of nuclear time delays. For supercritical systems the situation is significantly different. Figure 1 compares the anisotropy coefficient $a^{1}$ for the systems $\mathrm{U}-\mathrm{U}$ and $\mathrm{Pb}-\mathrm{Pb}$ in dependence on the nuclear time delay $T$, where an integration over a large window of electron and positron energies has been performed. The oscillation structures would appear more pronounced for smaller energy windows. Nevertheless, the anisotropy coefficient of subcritical systems would remain positive definite. In contrast, the anisotropy coefficient of the supercritical system $U+U$ becomes clearly negative, indicating a large difference between $\kappa=-1$ and $\kappa=+1$ contributions caused by the presence of spontaneous pos- 


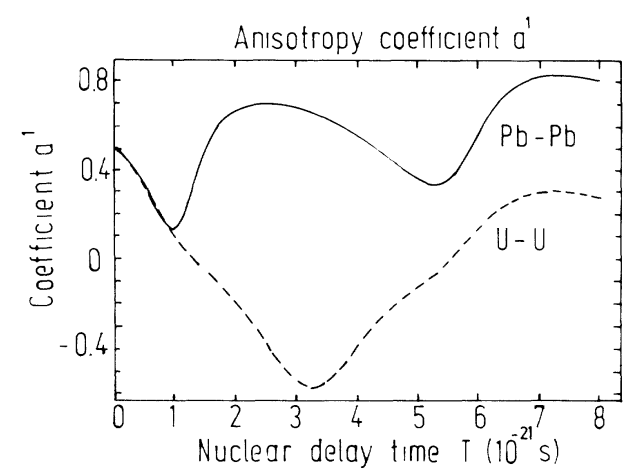

FIG. 1. Anisotropy coefficient $a^{1}$ for central U-U (dashed lines) and $\mathrm{Pb}-\mathrm{Pb}$ (full lines) collisions at $E_{\text {lab }}=6.2 \mathrm{MeV} /$ nucleon. The excitation rates have been integrated over a double energy window $102<E_{e^{-}}, E_{e^{+}}<588 \mathrm{keV}$. All values refer to the center-of-mass system.

itron production. This effect occurs already at relatively small delay times $T \approx 2 \times 10^{-21} \mathrm{~s}$. Presently available experimental data on deep inelastic $U+U$ collisions have given evidence for nuclear delay times up to the order of $T \approx(1-2) \times 10^{-21} \mathrm{~s}$ at energies well above the Coulomb barrier. ${ }^{16,17}$ In the present calculations we have employed $6.2 \mathrm{MeV} /$ nucleon, which would be favorable in view of the reduced nuclear background. It remains to be investigated whether a significant fraction of collisions at such low energies leads to comparable delay times. We have checked, however, that the angular correlations at $7.5 \mathrm{MeV} /$ nucleon qualitatively exhibit the same behavior.

A more realistic model for the nuclear reaction process has to account for a distribution of nuclear delay times $T$. As an example we use a Gaussian distribution function with mean $\bar{T}$ with width $\tau$. Figure 2 shows positron spectra in coincidence with electrons of definite energy, folded with the Gaussian time distribution in comparison with undelayed collisions, $T=0$. The chosen value of the parameter $\bar{T}=2 \times 10^{-21} \mathrm{~s}$ is the lower limit to yield a clear signal in the angular correlation. We note that the angular correlation coefficient $A$ always remains positive in the subcritical system. In contrast, the correlation coefficient $C$ of the supercritical system is enhanced, while the angular correlation coefficient $A$ turns out to be negative for a large region of positron energies. Both effects are a direct consequence of the spontaneous positron production mechanism, which continues to act while the electric field is static. Since the excitation rates involve a sum over products of four different amplitudes, which depend on the delay time $T$ in a complicated manner, it is difficult to understand the dependence of angular correlations on the nuclear delay time $T$ in terms of a simple model. Our calculations, however, have clearly revealed a qualitative difference in the behavior of subcritical and supercritical systems.

Whether this effect can be used as a signal for spon- (a)

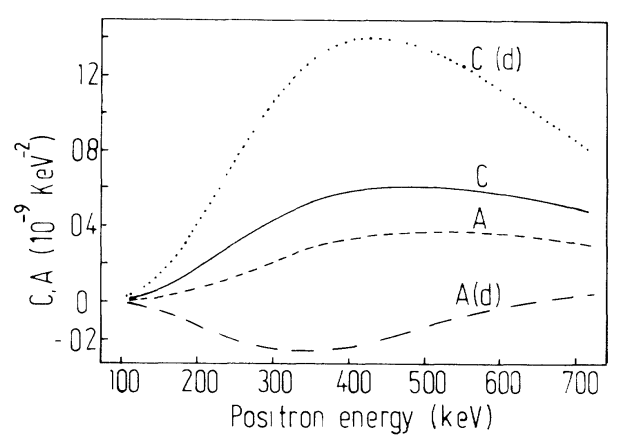

(b)

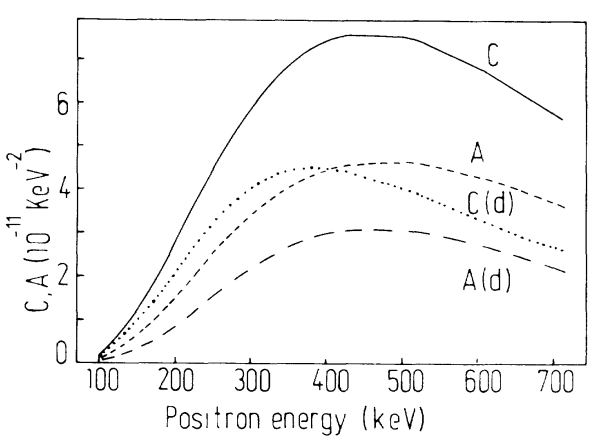

FIG. 2. Coefficients $C$ and $A$ [see Eq. (2)] for variable positron energies referring to an electron energy of $332 \mathrm{keV}$. The coefficient $S$ is not shown since it is negligibly small. Spectra for undelayed elastic collisions (coefficients $C$ and $A$ ) are compared to spectra which have been folded with a Gaussian distribution function of nuclear delay times [coefficients $C(d)$ and $A(d)$ ]. The chosen parameters for the Gaussian distribution are $\bar{T}=2 \times 10^{-21} \mathrm{~s}$ and $\tau=7 \times 10^{-22} \mathrm{~s}$. The calculations refer to central $(b=0)$ collisions at $E_{\text {lab }}=6.2 \mathrm{MeV} /$ nucleon. (a) U$\mathrm{U}$; (b) $\mathrm{Pb}-\mathrm{Pb}$.

taneous positron production depends on the strength of the unavoidable background $K\left(\theta_{e^{+}} e^{-}\right)$originating from internal pair conversion (IPC) of excited nuclear states. The background intensity can be determined by folding the measured $\gamma$-ray spectra with the well-known pair conversion coefficients. ${ }^{18}$ Using the $\gamma$-ray spectra of Ref. 17 we found that IPC is negligible against quasimolecular $e^{+} e^{-}$pair production in supercritical quasielastic collisions of bare nuclei. In deep-inelastic collisions both contributions are of comparable magnitude. Nevertheless, one may hope to discriminate between these processes by employing their different angular distributions. As an estimate one can assume the most unfavorable case, namely, all nuclear transitions to be of multipolarity $E 1$, and fold the IPC rates with the corresponding angular distribution. ${ }^{19}$ (Some uncertainties arise from the unavailability of angular differential conversion coefficients beyond the Born approximation.) Employing this procedure and assuming IPC to take place after symmetric fission we calculated the sum of dynamically created $e^{+} e^{-}$pairs and contributions from IPC for a time-delayed $\left(T=2 \times 10^{-21} \mathrm{~s}\right)$ collision of bare $\mathrm{U}$ nuclei 
at $7.5 \mathrm{MeV} /$ nucleon. For positron energies close to 400 $\mathrm{keV}$ the angular correlation of quasimolecular pairs and the weak suppression of IPC in the antiparallel emission hemisphere $\left(\pi / 2 \leq \theta_{e^{+} e^{-}} \leq \pi\right)$ suffice to guarantee a ratio

$$
R=\frac{P_{e^{+} e^{-}}-(\pi)}{P_{e^{+} e^{-}}(\pi / 2)}=\frac{C-A+K(\pi)}{C+K(\pi / 2)} \approx 1.1 .
$$

In comparison, $R$ would have a value close to 0.5 in the absence of spontaneous positron production.

In summary, we have shown that angular correlations of electron-positron pairs may be used as an additional instrument in the search for traces of spontaneous positron creation in heavy-ion collisions. For each experiment also the corresponding $\gamma$-ray spectrum has to be determined to calculate the influence of nuclear IPC, and the assumptions for the estimate made above have to be checked. To get a clear signal in the form of a ratio $P_{e^{+} e^{-}}(\pi) / P_{e^{+} e^{-}}(\pi / 2)>1$ one needs collisions of bare ions with a time delay of about $T \approx 2 \times 10^{-21} \mathrm{~s}$.

${ }^{1}$ W. Greiner, B. Müller, and J. Rafelski, Quantum Electrodynamics of Strong Fields (Springer-Verlag, Berlin, 1985).

${ }^{2}$ J. Reinhardt, B. Müller, and W. Greiner, Phys. Rev. A 24, 103 (1981).

${ }^{3}$ J. S. Greenberg and P. Vincent, in Treatise on Heavy-Ion Science, edited by D. A. Bromley (Plenum, New York, 1985), Vol. 5.

${ }^{4}$ M. A. Herath-Banda, W. Koenig, B. Martin, F. Güttner, H. Skapa, J. Soltani, K. Dworschak, F. Bosch, Ch. Kozhuharov, and A. V. Ramayya, Phys. Rev. A 33, 861 (1986).

${ }^{5}$ H. Backe, L. Handschug, F. Hessberger, E. Kankeleit, L. Richter, F. Weik, R. Willwater, H. Bokemeyer, P. Vincent, Y. Nakayama, and J. S. Greenberg, Phys. Rev. Lett. 40, 1443 (1978); C. Kozhuharov, P. Kienle, E. Berdermann, H. Bokemeyer, J. S. Greenberg, Y. Nakayama, P. Vincent, H. Backe, L. Handschug, and E. Kankeleit, Phys. Rev. Lett.
42, 376 (1979).

${ }^{6} \mathrm{~J}$. Schweppe, A. Gruppe, K. Bethge, H. Bokemeyer, T. Cowan, H. Folger, J. S. Greenberg, H. Grein, S. Ito, R. Schulé, D. Schwalm, K. E. Stiebing, N. Trautmann, P. Vincent, and M. Waldschmidt, Phys. Rev. Lett. 51, 2261 (1983); M. Clemente, E. Berdermann, P. Kienle, H. Tsertos, W. Wagner, C. Kozhuharov, F. Bosch, and W. Koenig, Phys. Lett. 137B, 41 (1984); T. Cowan, H. Backe, M. Begemann, K. Bethge, H. Bokemeyer, H. Folger, J. S. Greenberg, H. Grein, A. Gruppe, Y. Kido, M. Klüver, D. Schwalm, J. Schweppe, K. E. Stiebing, N. Trautmann, and P. Vincent, Phys. Rev. Lett. 54, 1761 (1985); H. Tsertos, E. Berdermann, F. Bosch, M. Clemente, P. Kienle, W. Koenig, C. Kozhuharov, and W. Wagner, Phys. Lett. 162B, 273 (1985); P. Kienle, J. Phys. Soc. Jpn. 54, Suppl. II, 549 (1985).

${ }^{7}$ J. Rafelski, B. Müller, and W. Greiner, Z. Phys. A 285, 49 (1978).

${ }^{8} \mathrm{~J}$. Reinhardt, U. Müller, B. Müller, and W. Greiner, Z. Phys. A 303, 173 (1981).

${ }^{9}$ T. de Reus, J. Reinhardt, B. Müller, W. Greiner, G. Soff, and U. Müller, J. Phys. B 17, 615 (1984).

${ }^{10}$ E. Kankeleit, Nukleonika 25, 253 (1980).

${ }^{11}$ M. A. Herath-Banda, A. V. Ramayya, C. F. Maguire, F. Güttner, W. Koenig, B. Martin, B. Povh, H. Skapa, and J. Soltani, Phys. Rev. A 29, 2429 (1984).

12 J. D. Molitoris, R. Anholt, W. E. Meyerhof, O. K. Baker, S. Andriamonje, and E. Morenzoni, Z. Phys. A 323, 127 (1986).

${ }^{13}$ O. Graf, J. Reinhardt, T. de Reus, B. Müller, W. Greiner, and G. Soff, Z. Phys. A 328, 81 (1987).

${ }^{14}$ K. Rumrich, Diploma thesis, Universität Frankfurt, 1987 (to be published).

${ }^{15}$ G. Soff, J. Reinhardt, B. Müller, and W. Greiner, Phys. Rev. Lett. 43, 1981 (1979).

${ }^{16}$ M. Nessi, Ch. Stoller, E. Morenzoni, W. Wölfli, W. E. Meyerhof, J. D. Molitoris, E. Grosse, and Ch. Michel, Phys. Rev. C 36, 143 (1987).

${ }^{17}$ R. Krieg, E. Bozek, U. Gollerthan, E. Kankeleit, G. KlotzEngmann, M. Krämer, U. Meyer, H. Oeschler, and P. Senger, Phys. Rev. C 34, 562 (1986).

${ }^{18}$ P. Schlüter, G. Soff, and W. Greiner, Z. Phys. A 286, 149 (1978).

${ }^{19}$ M. E. Rose, Phys. Rev. 76, 678 (1949). 